\title{
Monitoring and comorbidities in patients with chronic hepatitis B currently treated with nucleos(t)ide analogs
}

\author{
Spyros Siakavellas a, John Goulis ${ }^{b}$, Spilios Manolakopoulos ${ }^{a, c}$, Christos Triantos $^{d}$, Nikolaos Gatselise, \\ Eva Tsentemidou ${ }^{b}$, Hariklia Kranidiotic, Konstantinos Zisimopoulos ${ }^{d}$, Christos Tsoulas ${ }^{f}$, \\ George Dalekos', George Papatheodoridis ${ }^{a}$
}

Medical School of National and Kapodistrian University of Athens, "Laiko" General Hospital of Athens; Medical School of Aristotle University of Thessaloniki; Medical School of National and Kapodistrian University of Athens, Hippokratio General Hospital; University Hospital of Patras, Rio; General University Hospital of Larissa; Gilead Sciences Hellas, Greece

\section{Abstract}

\begin{abstract}
Background Long-term monotherapy with nucleos(t)ide analogs (NAs) represents the treatment option for the majority of patients with chronic hepatitis $\mathrm{B}(\mathrm{CHB})$, an aging population with a greater likelihood of comorbidities. We assessed the prevalence of concurrent non-hepatic diseases and the safety monitoring in a large cohort of CHB patients receiving NAs and their potential impact on disease outcomes.

Methods We included 500 consecutive CHB patients from 5 major tertiary Greek centers, under long-term therapy with an NA. Epidemiological/clinical characteristics and data on concomitant disease, drug use and investigations ordered were collected.

Results The mean age was 58 years and 66\% were male. Most patients were receiving tenofovir disoproxil fumarate (TDF, 60\%) or entecavir (ETV, 37\%) monotherapy. Decompensated cirrhosis at baseline was present in $10 \%$, while hepatocellular carcinoma (HCC) under therapy developed in 21 patients. The median duration of total NA therapy was 56 and of latest therapy 42 months. The most common (prevalence $>10 \%$ ) comorbidities were hypertension (28\%), non-HCC cancer(s) (12\%), and diabetes (11\%). Patients with a longer duration of latest therapy ( $\geq 4$ vs. $<4$ years) were older (mean age: 58 vs. 56 years, $\mathrm{P}=0.004)$, had more frequent history of prior use of $\mathrm{NA}(\mathrm{s})(53 \%$ vs. $35 \%, \mathrm{P}<0.001)$, and less frequent liver decompensation ( $5 \%$ vs. $13 \%, \mathrm{P}=0.008)$ and non-HCC cancers ( $8 \%$ vs. $15 \%, \mathrm{P}=0.020)$. HCC developed more frequently in patients with than in those without diabetes ( $11 \%$ vs. $3 \%, \mathrm{P}=0.022)$.

Conclusion Greek CHB patients currently treated with NAs, almost exclusively ETV or TDF, are often older than 60 years, have several comorbidities, and thus require careful management.
\end{abstract}

Keywords Chronic hepatitis B, nucleos(t)ide analog, comorbidities, monitoring

Ann Gastroenterol 2020; 33 (6): 1-7

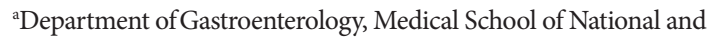
Kapodistrian University of Athens, "Laiko" General Hospital of Athens, Athens (Spyros Siakavellas, Spilios Manolakopoulos, George Papatheodoridis); $4^{\text {th }}$ Department of Internal Medicine, Medical School of Aristotle University of Thessaloniki (John Goulis, Eva Tsentemidou); ${ }^{\text {c }}{ }^{\text {nd }}$ Department of Internal Medicine, Medical School of National and Kapodistrian University of Athens, Hippokratio General Hospital, Athens (Spilios Manolakopoulos, Hariklia Kranidioti); ${ }^{\mathrm{d} D i v i s i o n}$ of Gastroenterology, Department of Internal Medicine, University Hospital of Patras, Rio (Christos Triantos, Konstantinos Zisimopoulos); ${ }^{\text {DDepartment }}$ of Medicine and Research Laboratory of Internal Medicine, National Expertise Center of Greece in Autoimmune Liver Diseases, General University Hospital of Larissa (Nikolaos Gatselis, George Dalekos); ${ }^{\mathrm{f}}$ Medical Department, Gilead Sciences Hellas (Christos Tsoulas), Greece

Conflict of Interest: This study was supported by an unrestricted grant from Gilead Sciences Hellas

Correspondence to: George V. Papatheodoridis, MD, PhD, Director of Department of Gastroenterology, School of Medicine, National and Kapodistrian University of Athens, Laiko General Hospital of Athens, 17 Agiou Thoma St, 11527 Athens, Greece, e-mail: gepapath@med.uoa.gr

Received 10 May 2020; accepted 25 June 2020;

published online 10 July 2020

DOI: https://doi.org/10.20524/aog.2020.0525

\section{Introduction}

Chronic infection with hepatitis B virus (HBV) constitutes an important global public health problem [1]. While its prevalence has been decreasing as a result of comprehensive vaccination programs and improvement in socioeconomic status, the number of cases with chronic $\mathrm{HBV}$ infection is not decreasing dramatically because of the increasing global population $[1,2]$. In addition, given the increasing age of patients with $\mathrm{HBeAg}$-positive or HBeAg-negative chronic hepatitis $\mathrm{B}(\mathrm{CHB})$, the active phases of chronic HBV infection, increases in morbidity and mortality are expected in the near future [1]. A significant development contributing to the better management of this disease has been the introduction of nucleos(t)ide analogs (NAs). The potent longterm inhibition of HBV replication by NAs has repeatedly been shown to improve all clinical outcomes in CHB patients. Moreover, NAs have been shown to have an advantageous tolerance and safety profile, very favorable compared to pegylated interferon alpha, the other therapeutic option for CHB patients [3,4]. Furthermore, they comprise the only choice for a variety of patient 
subgroups, such as those with advanced cirrhosis, indications for immunosuppression, or extrahepatic manifestations $[3,5,6]$. Therefore, the vast majority of $\mathrm{CHB}$ patients are currently treated with NAs. There are quite a few NAs approved for use in CHB, but the use of those with a low genetic barrier to virologic resistance, such as lamivudine, adefovir dipivoxil and telbivudine, is not recommended. Thus, the currently recommended NAs for naïve $\mathrm{CHB}$ patients are entecavir (ETV), tenofovir disoproxil fumarate (TDF) and tenofovir alafenamide (TAF), while TDF and TAF are the only options for patients who have previous exposure and are particularly resistant to lamivudine or any other nucleoside analog (telbivudine, ETV) [1].

Therapeutic administration of ETV, TDF or TAF provides an excellent option for the long-term management of $\mathrm{CHB}$, with the caveat that it rarely leads to HBV surface antigen (HBsAg) clearance or "functional cure". Therefore, treatment is usually continued indefinitely, making careful long-term monitoring necessary $[1,7,8]$. Given that the $\mathrm{CHB}$ patient population is gradually aging, additional health issues may either coexist from the onset of therapy or accumulate on the way. Thus, current $\mathrm{CHB}$ patients treated with NAs have a greater likelihood of coexisting comorbidities and more contemporaneous use of other pharmaceutical products.

Regarding the safety monitoring of $\mathrm{CHB}$ patients treated with NAs, baseline and periodical on-therapy laboratory tests are recommended, with special focus on renal function [1]. Although long-term NA administration is generally considered to have minimal effects on kidney function and bone mineral density, based mostly on long-term ETV and TDF data, there have been reports of progressive worsening of renal function and bone mineral density as well as of renal tubulopathy and even Fanconi syndrome in patients on TDF [10-12]. On the other hand, recent phase III trials have shown that, in terms of renal and bone laboratory parameters, TAF has a more favorable safety profile than TDF $[13,14]$. Currently, selection of ETV or TAF instead of TDF, as well as switching from TDF to ETV or TAF, is recommended for CHB patients with or at high risk for renal or bone disorders, with TAF being the only recommended option for lamivudine-experienced patients [1].

In view of the aforementioned issues, we decided to conduct a study in $\mathrm{CHB}$ patients receiving NAs with the following objectives: a) to assess the prevalence of underlying concurrent non-hepatic medical issues and their potential impact on the liver disease course and outcomes; and b) to observe the safety monitoring in real-life situations at tertiary hepatology centers in our country.

\section{Patients and methods}

\section{Patient population}

A retrospective analysis was conducted in a cohort of 500 consecutive $\mathrm{CHB}$ patients receiving NAs and visited the outpatient liver clinics of 5 tertiary Hepatology Centers in our country between January and April 2017; 100 patients from each center were included. All patients fulfilled the national indications for treatment with NAs. In particular, all patients had chronic HBV infection, defined by HBsAg seropositivity for at least 6 months before the onset of NA therapy, and a) HBV DNA $>20,000 \mathrm{IU} / \mathrm{mL}$ and alanine aminotransferase (ALT) activity higher than twice the upper limit of normal; or b) HBV DNA $>2000 \mathrm{IU} / \mathrm{mL}$ and/or elevated ALT and at least moderate necroinflammatory activity and/or fibrosis stage; or c) cirrhosis and detectable HBV DNA, regardless of ALT levels. The only exclusion criterion was the presence of coinfection with hepatitis $\mathrm{D}$ virus, hepatitis $\mathrm{C}$ virus or human immunodeficiency virus. All patients were treated with $\mathrm{NA}(\mathrm{s})$ at the recommended daily dosage, according to the summary product characteristics of each medication, while the recommended dosage adjustments were applied in patients with glomerular filtration rate (eGFR) $<50 \mathrm{~mL} / \mathrm{min}$, as estimated by serum creatinine levels, and patient characteristics using the Cockcroft-Gault equation.

All relevant demographic, clinical and pharmacologic information, in addition to data regarding concomitant diseases and investigations ordered, were retrospectively collected from the patient records and entered into a specifically designed database. In particular, the parameters recorded were: age, nationality, height, weight, body mass index (BMI), alcohol and smoking habits, diagnosis date, previous (including prior lamivudine use) and latest treatments (with initiation and end dates if applicable), history of liver biopsy, liver stiffness measurements, presence of decompensated cirrhosis at onset of NA treatment, hepatocellular carcinoma (HCC) development during NA treatment, concomitant (non-hepatic) diseases, other medications taken, tests performed, and frequency of relevant investigations while on NA therapy. At least moderate fibrosis or compensated cirrhosis was diagnosed by either liver biopsy, or liver stiffness $>9$ or $>14$ $\mathrm{kPa}$ by reliable liver elastography, respectively. Decompensated cirrhosis was diagnosed in patients with development of ascites, history of hepatic encephalopathy or variceal bleeding and/or jaundice (total bilirubin $>3 \mathrm{mg} / \mathrm{dL}$ ) of non-obstructive cause. All patients were informed regarding the anonymous use of their personal data and consented to their inclusion in the study.

\section{Statistical analysis}

The SPSS and GraphPad statistical software programs were used for the analysis. Quantitative variables were expressed as mean \pm standard deviation (SD) or as median (range) values, depending on whether they had normal or abnormal distribution, respectively. Categorical variables were compared using the chisquared test, whereas the $t$-test and ANOVA testing (where more than 2 groups were compared) were used for comparisons between quantitative variables. Spearman's $r$-test was used to assess correlations between quantitative variables. In all cases, an alpha level of $<0.05$ was considered to be statistically significant.

\section{Results}

\section{Patient characteristics}

The main patient- and disease-related characteristics are shown in Table 1 . Of the 500 patients, $46 \%$ were older than 
60 years. The majority of patients $(n=407,81 \%)$ were Greek and a considerable minority were immigrants living in Greece and born in Albania ( $\mathrm{n}=59,12 \%)$. Interestingly, the Greek patients in our cohort were significantly older than those born in Albania or other countries $(60.5 \pm 14.2$ vs. $45.5 \pm 11.0$ years, $\mathrm{P}<0.001)$. Greek patients also tended to have higher weight and BMI, but this difference may be attributed to their being older, as it did not remain significant after adjustment for age. There were no significant differences in the basic demographic characteristics among the patients of the different tertiary centers participating in the study (data not shown).

As TAF was not available in Greece at the time of patients' enrolment in this study, most patients were receiving either TDF (60\%) or ETV (37\%) monotherapy, while only a small minority were receiving combination treatment with both TDF and ETV. The median duration of antiviral therapy with the last NA was 42 months (range 1-186), while the median duration of the total antiviral therapy was 56 months (range 1-212). A substantial portion of the patients of this cohort were lamivudine-experienced (31\%), while two thirds of this subgroup had documented lamivudine resistance. In the lamivudine-experienced group, $85 \%$ of the patients were receiving TDF, while this percentage rose to about $90 \%$ in the subgroup of patients with documented lamivudine resistance.

Before treatment initiation, liver biopsy had been performed in 124 (25\%) patients, while liver stiffness measurement by FibroScan or Shear-Wave elastography was available in another $210(42 \%)$ cases. At least moderate fibrosis was detected in $67 \%$ and compensated cirrhosis in $21 \%$ of 334 patients with pretreatment assessment of fibrosis. Of our study population, 48 (10\%) patients had decompensated cirrhosis at the onset of the last antiviral therapy, while no new case of liver decompensation was observed after the onset of the last NA. HCC was diagnosed in 21 (4\%) patients during on-treatment follow up. No patient died or underwent liver transplantation. Virological remission defined by undetectable serum HBV DNA was observed in 96\% and biochemical remission defined by normal ALT in $92 \%$ of 422 patients who had completed at least 12 months of last NA therapy.

We also examined our patient characteristics in relation to the duration of treatment with their latest NA, which also defines the

Table 1 Clinical and demographic characteristics of 500 consecutive patients with chronic hepatitis B receiving nucleos(t)ide analogs

\begin{tabular}{|c|c|}
\hline Characteristics & Value \\
\hline Age, years & $57.7 \pm 14.9(20-88)$ \\
\hline Male sex & $329(66 \%)$ \\
\hline Body mass index, $\mathrm{kg} / \mathrm{m}^{2}$ & $26.4 \pm 4.3(15.6-43.0)$ \\
\hline $\begin{array}{l}\text { Place of birth } \\
\text { Greece } \\
\text { Albania } \\
\text { Other country }\end{array}$ & $\begin{array}{c}407(81 \%) \\
59(12 \%) \\
34(7 \%)\end{array}$ \\
\hline $\begin{array}{l}\text { Alcohol consumption } \\
\text { None } \\
\text { Social use }(<40 / 20 \mathrm{~g} / \text { day for males/females }) \\
\text { Abuse ( } \geq 40 / 20 \mathrm{~g} / \text { day for males/females })\end{array}$ & $\begin{array}{c}375(75 \%) \\
105(21 \%) \\
20(4 \%)\end{array}$ \\
\hline $\begin{array}{l}\text { Smoking } \\
\text { Never smoked } \\
\text { Former smoker } \\
\text { Current smoker }\end{array}$ & $\begin{array}{l}353(71 \%) \\
52(10 \%) \\
95(19 \%)\end{array}$ \\
\hline $\begin{array}{l}\text { Latest antiviral treatment } \\
\text { Tenofovir disoproxil fumarate (TDF) } \\
\text { Entecavir (ETV) } \\
\text { TDF+ETV }\end{array}$ & $\begin{array}{c}301(60 \%) \\
185(37 \%) \\
14(3 \%)\end{array}$ \\
\hline Duration of last antiviral treatment, months & $42(1-168)$ \\
\hline Previous antiviral treatment, Yes & $213(43 \%)$ \\
\hline Total duration of antiviral treatment, months & $56(1-212)$ \\
\hline Patients with lamivudine experience & $156(31 \%)$ \\
\hline Patients with lamivudine resistance & $104(21 \%)$ \\
\hline At least moderate fibrosis before the onset of last therapy & $224 / 334^{*}(67 \%)$ \\
\hline Compensated cirrhosis before the onset of last therapy & $70 / 334^{*}(21 \%)$ \\
\hline Decompensated cirrhosis at the onset of last therapy & $48(10 \%)$ \\
\hline Hepatocellular carcinoma diagnosed during therapy & $21(4 \%)$ \\
\hline
\end{tabular}


time when treatment with that NA began. Using 4 years (close to the mean value of 45 months for latest treatment duration) as cutoff, our patients were classified into those with old ( $>4$ years) and recent ( $\leq 4$ years) treatment onset. The main differences in the patient characteristics in relation to their duration of treatment are summarized in Fig. 1. In particular, patients with old NA therapy onset were older (mean age: 58 vs. 56 years, $\mathrm{P}=0.004$ ), more likely to have received another NA in the past (53\% vs. $35 \%$, $\mathrm{P}<0.001)$, more likely to have undergone liver biopsy at baseline ( $39 \%$ vs. $14 \%, \mathrm{P}<0.001$ ), less likely to have decompensated disease at baseline ( $5 \%$ vs. $13 \%, \mathrm{P}=0.008)$, and less likely to have concurrent non-hepatic neoplasia ( $8 \%$ vs. $15 \%, \mathrm{P}=0.020$ ).

\section{Comorbidities}

The most common (prevalence $>2 \%$ ) concurrent conditions observed in our patient cohort can be seen in Table 2 and Fig. 2. The most commonly encountered comorbidities in our study were arterial hypertension $(\mathrm{n}=139,28 \%)$, non-hepatic malignancy $(n=61,12 \%)$, diabetes mellitus $(n=56,11 \%)$, rheumatologic diseases $(n=51,10 \%)$, and thyroid disorders $(n=47,9 \%)$. Metabolic bone disorder and severe renal impairment, which constitute a concern in patients receiving NAs, were present in $8 \%$ and $3 \%$ of patients, respectively. Of particular interest is the fact that $11(85 \%)$ of the 13 patients with renal failure (estimated creatinine clearance $<30 \mathrm{~mL} / \mathrm{min}$ ) were receiving ETV, while 2 were on TDF, albeit under very close renal monitoring. Moreover, of the 40 patients with metabolic bone disorder (including both osteopenia and osteoporosis), 75\% were on TDF and only $25 \%$ on ETV. Another interesting observation was the greater prevalence $(n=24,39 \%)$ of hematologic malignancies among the 61 patients with non-hepatic malignancies.

\section{Monitoring of patients}

In our cohort, patients were generally seen approximately every 6 months $(6.0 \pm 2.7)$, as recommended by our National

Table 2 Most common comorbidities in chronic hepatitis B patients under treatment with entecavir and/or tenofovir disoproxil fumarate

\begin{tabular}{lc}
\hline Comorbidity & Patients, $\mathrm{n}(\%)$ \\
\hline Hypertension & $139(27.8)$ \\
Non-hepatocellular malignancy & $61(12.2)$ \\
Diabetes mellitus & $56(11.2)$ \\
Rheumatologic disease & $51(10.2)$ \\
Thyroid disease & $48(9.4)$ \\
Obesity & $46(9.2)$ \\
Metabolic bone disease & $40(8.0)$ \\
Hyperlipidemia & $31(6.2)$ \\
Organ transplantation & $16(3.2)$ \\
Severe renal impairment & $13(2.6)$ \\
\hline
\end{tabular}

${ }^{\star}$ Estimated glomerular filtration rate $<30 \mathrm{~mL} / \mathrm{min}$ estimated by the Cockcroft-Gault equation
Guidelines for the management of $\mathrm{CHB}$, similar to those developed by the European Association for the Study of the Liver (EASL) [1]. The most common tests used for efficacy and safety monitoring of our patients, the proportion of patients undergoing each test and the frequency of each test can be seen in Table 3.

Across all 5 centers, a standard laboratory panel, including at least complete blood count, liver function tests and serum creatinine, was performed in more than $90 \%$ of patients almost every 6 months, while serum phosphate levels were also measured with similar frequency in $35 \%$ of all patients, or $56 \%$ of patients treated with TDF. Abdominal ultrasonography, often combined with alfa fetoprotein measurements, was performed

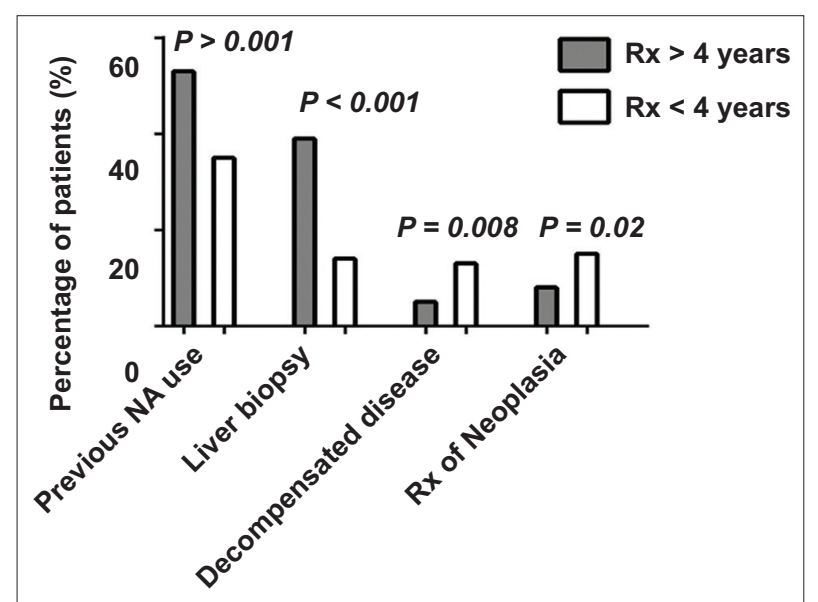

Figure 1 Main differences in characteristics of treated patients with chronic hepatitis B in relation to their duration of treatment ( $>4$ vs. $\leq 4$ years)

$N A$, nucleos(t)ide analog

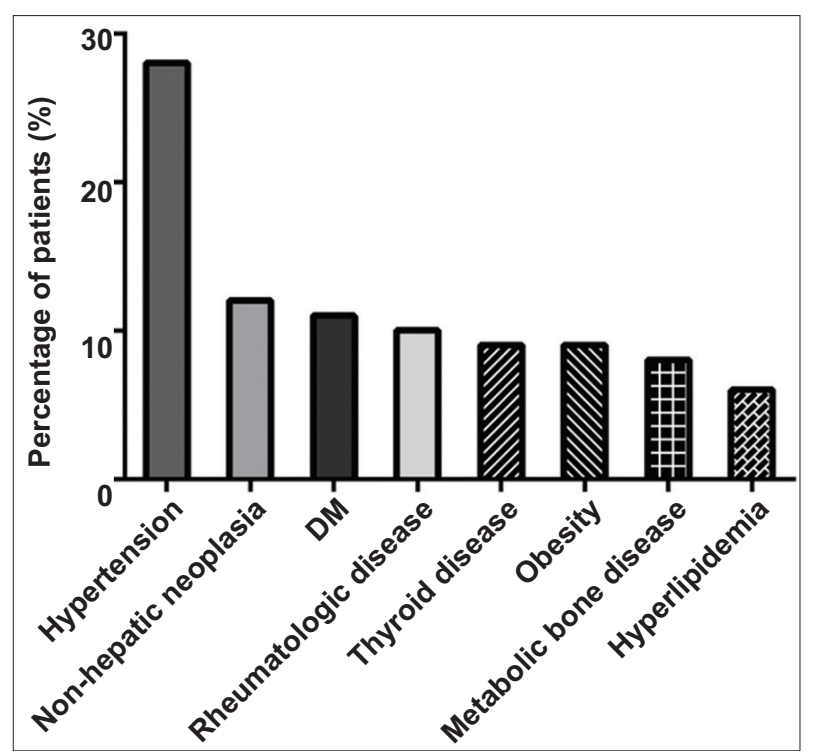

Figure 2 Prevalence of common comorbidities in patients with chronic hepatitis B under treatment with entecavir and/or tenofovir disoproxil fumarate

$D M$, diabetes mellitus 
approximately every 10 months in up to $89 \%$ of patients. The proportion of patients who underwent HCC surveillance at least with ultrasonography did not differ significantly between cases with or without pretreatment for compensated or decompensated cirrhosis (110/118 or $93 \%$ vs. $243 / 264$ or $92 \%)$, or between cases at low (PAGE-B $<10$ ) and medium-high HCC risk (PAGE-B $\geq 10$ ) ( $92 / 102$ or $90 \%$ vs. $329 / 367$ or $89 \%$ ). However, HCC surveillance was performed more frequently in patients at medium-high risk than in those with low HCC risk (mean frequency: 9.1 \pm 4.8 vs. $9.5 \pm 3.7$ months, $\mathrm{P}=0.002$ ), but it did not differ significantly between patients with or without pretreatment cirrhosis (mean frequency: $9.7 \pm 4.4$ vs. $11.4 \pm 4.5$ months, $\mathrm{P}=0.506$ ). It is worth mentioning that the tests performed less frequently than once annually were serum HBV DNA determination (91\% of patients), as well as liver stiffness in $46 \%$ and bone mineral density measurement in only $6 \%$ of patients.

\section{Disease outcome associated with presence of specific comorbidities}

There was a trend for decompensated cirrhosis to be more commonly present in obese than in non-obese $\mathrm{CHB}$ patients ( $17 \%$ vs. $9 \%, \mathrm{P}=0.068$ ). More importantly, HCC was found to have developed more often in diabetic $\mathrm{CHB}$ patients than in those without diabetes mellitus ( $11 \%$ vs. $3 \%, \mathrm{P}=0.022)$ (Fig. 3 ), more common in patients with pretreatment cirrhosis $(20 \%$ vs. $8 \%, \mathrm{P}<0.001)$. In addition, patients with HCC development were significantly older than those without (mean age: $69 \pm 11$ vs. $57 \pm 15$ years, $\mathrm{P}=0.004$ ) and were more likely to have had pretreatment for compensated $(5 / 70$ or $7.1 \%$ vs. $1 / 264$ or $0.4 \%, \mathrm{P}<0.001)$ or decompensated cirrhosis $(11 / 21$ or $52.4 \%$ vs. $37 / 479$ or $7.7 \%, \mathrm{P}<0.001)$. HCC patients also had a higher mean PAGE-B score $(19 \pm 4$ vs. $14 \pm 6, \mathrm{P}<0.001)$, while the 2 groups did not differ significantly in relation to patients' place of birth, sex, BMI, alcohol or smoking habits. None of the patients who developed HCC had low risk $(<10)$ according to PAGE-B score.

Table 3 Most common tests used for the monitoring of chronic hepatitis $B$ patients under treatment with entecavir and/or tenofovir disoproxil fumarate

\begin{tabular}{llc}
\hline Test & $\begin{array}{l}\text { Proportion } \\
\text { of patients }\end{array}$ & $\begin{array}{c}\text { Frequency of test in } \\
\text { months (mean } \pm \text { SD, range) }\end{array}$ \\
\hline
\end{tabular}

\begin{tabular}{lcc}
\hline Liver function tests & $100 \%$ & $6.1 \pm 3.1(2-12)$ \\
Complete blood count & $95 \%$ & $6.2 \pm 2.9(2-12)$ \\
Serum HBV DNA & $92 \%$ & $15.8 \pm 9.2(3-48)$ \\
Serum creatinine & $94 \%$ & $6.2 \pm 2.8(1-12)$ \\
Serum phosphate & $35 \% *$ & $6.3 \pm 2.9(3-12)$ \\
Alfa fetoprotein & $69 \%$ & $10.0 \pm 4.2(3-24)$ \\
Liver ultrasound & $89 \%$ & $10.2 \pm 4.5(3-24)$ \\
Liver elastography & $46 \%$ & $16.5 \pm 6.8(3-60)$ \\
Bone mineral density scan & $6 \%$ & $14.5 \pm 4.9(12-24)$ \\
\hline *56\% of patients treated with tenofovir disoproxil fumarate \\
HBV, hepatitis B virus; SD, standard deviation
\end{tabular}

\section{Discussion}

In the current study, we provide evidence from real-life data that $\mathrm{CHB}$ patients currently treated with NAs are an aging population, as almost $50 \%$ are close to or older than 60 years. Thus, they may exhibit a variety of coexisting non-hepatic diseases. These concurrent medical issues may potentially impact the liver disease course and pose significant challenges regarding their management.

According to our results, $\mathrm{CHB}$ patients seen and treated in Greece nowadays comprise 2 distinct subgroups. The largest subgroup includes the indigenous Greek patients, who tend to be older as chronic HBV infection has almost disappeared in young Greeks as a result of the universal adoption of neonate $\mathrm{HBV}$ vaccination during the last 20 years. On the other hand, there is a smaller but growing subgroup that includes mostly younger $\mathrm{CHB}$ patients who immigrated to Greece from countries with a higher HBsAg prevalence, such as Albania. This finding is in accordance with the changing HBV epidemiology in Greece that has already been reported in previous studies $[15,16]$. Thus, while HBV prevalence is decreasing in Greece, chronic HBV infection is not a medical disease that will disappear soon, and the pool of $\mathrm{CHB}$ patients receiving NAs is only expected to increase.

The NAs used in Greece over the last years were almost exclusively ETV and TDF, as TAF has only recently become available. Given that almost one third of our patients were lamivudine-experienced and one fifth had documented resistance to lamivudine, it seems that there is a substantial subgroup of $\mathrm{CHB}$ cases that should optimally be treated with TDF. However, the presence of comorbidities may raise valid concerns regarding the use of TDF. While severe renal impairment was not commonly encountered in our cohort, bone mineral density disorders including osteopenia represented the sixth most commonly observed concomitant medical condition. In addition, $46 \%$ of the patients in our study were over 60 years old, the age cutoff recommended by the latest EASL guidelines to be used for selection of patients who may benefit by avoiding TDF. Considering all the above, TAF, the newer HBV therapeutic option, seems to be quite

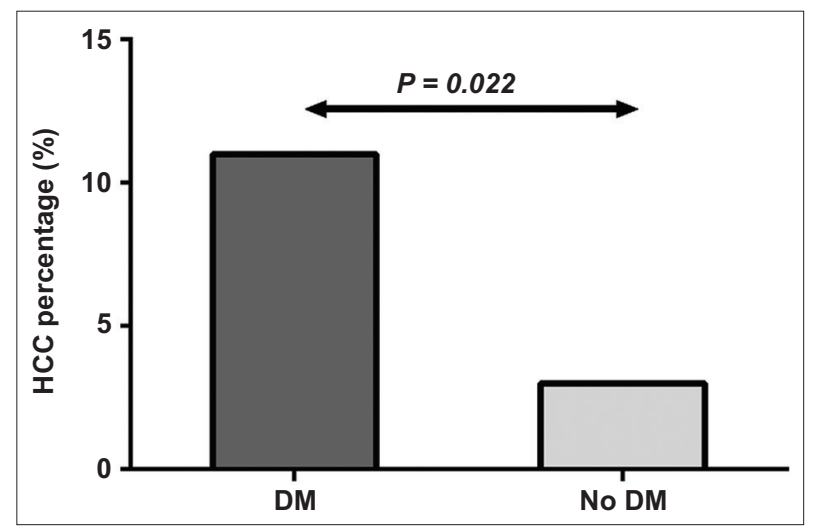

Figure 3 Proportion of treated chronic hepatitis B patients who developed hepatocellular carcinoma (HCC) in relation to the presence of diabetes mellitus (DM) 
helpful in the management of aging CHB patients, who often have a history of lamivudine exposure, and may be at risk of developing NA-related or unrelated bone or kidney disorders.

The prevalence of comorbidities encountered in our cohort does not seem to differ from that expected in the general Greek population of similar age groups [17-20]. As expected, hypertension and diabetes mellitus were the most common comorbidities observed in our $\mathrm{CHB}$ patients, who had a mean age close to 60 years. The high proportion of hematologic malignancies in the non-hepatic neoplasia group may be attributed to the great number of referrals from a busy hematology department of the hospital of one of the tertiary centers, as well as to the known frequent HBV reactivation in patients with hematologic malignancies [21]. On the other hand, it should be noted that HBV infection has been associated with increased risk for several types of cancers, including hematologic malignancies [22].

Regarding the differences observed between patients with old and recent treatment onset, the different age was selfexplanatory, whereas the higher likelihood of a history of prior exposure to another NA and of having undergone liver biopsy in the older treatment onset subgroup reflects the changes in clinical practice over time. It is clear that more patients receiving ETV and mostly TDF had been treated with another NA in the past, mostly with lamivudine, as the $a b$ initio use with ETV or TDF became standard practice in the last decade. Similarly, liver biopsy was a standard procedure for the majority of CHB patients before the onset of therapy in the past, while noninvasive methods, mainly liver elastography, have been widely used for the assessment of the severity of liver fibrosis over the last 5-10 years in Greece. Interestingly, the higher duration of suppression of HBV replication was associated with a lower prevalence of non-hepatic neoplasia, implying that HBV might also be somehow implicated in extrahepatic carcinogenesis, perhaps via dysregulation of the host's immune responses. However, such a finding needs confirmation as it might also represent a type I error. The higher proportion of patients with decompensated cirrhosis before treatment among those who started NA therapy more recently was also interesting and may reflect changes in the stages of chronic HBV patients at initial diagnosis in our country. Non-cirrhotic patients with earlier stages of fibrosis can be diagnosed only though asymptomatic screening, which is probably decreasing, while symptomatic patients who develop decompensated cirrhosis seek medical advice, which leads to diagnosis and immediate treatment.

Monitoring of treated $\mathrm{CHB}$ patients in tertiary centers in our country was found to be generally in line with the recommendations put forth by our National Association and EASL [1]. The main clear discrepancy was related to the frequency of serum HBV DNA determinations, repeated at a mean interval of 16 months, whereas the recommended interval is every 6-12 months. This is probably related to our national state insurance policy, as HBV DNA was not reimbursed until October 2017 and was available for free in very few public hospitals with very long waiting times. Given that the cost of HBV DNA testing in private laboratories was high, repeat testing was not often performed, mainly because of financial constraints. Bone mineral density measurements and liver elastography were also repeated less than once annually, but there have been no clear recommendations regarding the need and the frequency of these tests for CHB patients under long-term NA therapy. Moreover, gastroenterologists, who often follow CHB patients in Greece, may not be as familiar or comfortable managing osteopenia and osteoporosis, and even tend to underestimate and therefore not to monitor these conditions. The latter suggestion may also be related to the finding that $75 \%$ of our $\mathrm{CHB}$ patients with bone mineral disorders were receiving TDF, whose avoidance is currently recommended in such patients [1]. Therefore, appropriate close follow up is imperative to ensure the optimal management of these cases. Finally, facilities for liver stiffness measurements are not readily available throughout Greece and the examination remains currently out of the national health reimbursement list. Given these obstacles, the frequency of liver elastography monitoring observed in our study could even be considered as satisfactory.

The most significant impact on the liver disease outcomes from the presence of comorbidities in our study was the

\section{Summary Box}

\section{What is already known:}

- Long-term monotherapy with a nucleos(t)ide analog (NA) of high genetic barrier represents the treatment option for the vast majority of chronic hepatitis $\mathrm{B}(\mathrm{CHB})$ patients

- Treatment with a NA is usually given long-term, perhaps indefinitely, making careful long-term monitoring necessary

- Current CHB patients treated with NAs have an increased likelihood of coexisting comorbidities and increased use of comedications

\section{What the new findings are:}

- $\mathrm{CHB}$ patients currently treated with NAs in Greece are an aging population, as almost 50\% are close to or older than 60 years, and often have several comorbidities, thus requiring careful management

- Higher duration of inhibition of hepatitis B virus (HBV) replication is associated with a lower prevalence of non-hepatic neoplasia

- Monitoring of treated $\mathrm{CHB}$ patients in tertiary centers in Greece is in line with the EASL recommendations, except for the low frequency of serum HBV DNA determinations

- The concomitant presence of diabetes mellitus in $\mathrm{CHB}$ patients is associated with an increased risk for hepatocellular carcinoma (HCC) development; therefore, a high degree of alertness is essential in this subgroup for prompt diagnosis of HCC development at an early stage 
association of diabetes mellitus with the development of HCC. Diabetes has been already recognized as an independent risk factor for HCC in previous studies [23,24], and it has reasonably been suggested that the presence of diabetes and obesity exerts additional nefarious effects on the liver, acting in a synergistic manner with the underlying chronic HBV infection. Moreover, recent studies further highlight this association, raising a high degree of alertness in $\mathrm{CHB}$ patients with diabetes mellitus, essential in order not to miss the early signs of HCC development [25,26].

In conclusion, $\mathrm{CHB}$ patients currently treated with NAs in Greece receive exclusively TDF and/or ETV as long-term maintenance therapy; this may raise safety and compliance issues [27]. They are often older than 60 years and have several comorbidities; they thus require careful management. The coexisting non-hepatic conditions may affect the optimal choice of their anti-HBV agent and their disease course. Therefore, close follow up for an extended period, as well as careful management of the existing comorbidities in collaboration with relevant specialties (such as rheumatology and diabetes specialists), are warranted in this setting, in order to ensure better long-term clinical outcomes.

\section{References}

1. European Association for the Study of the Liver. EASL 2017 Clinical Practice Guidelines on the management of hepatitis B virus infection. J Hepatol 2017;67:370-398.

2. Chen CL, Yang JY, Lin SF, et al. Slow decline of hepatitis B burden in general population: Results from a population-based survey and longitudinal follow-up study in Taiwan. J Hepatol 2015;63:354-363.

3. Lok AS, McMahon BJ, Brown RS Jr, et al. Antiviral therapy for chronic hepatitis B viral infection in adults: A systematic review and meta-analysis. Hepatology 2016;63:284-306.

4. Liaw YF. Impact of therapy on the outcome of chronic hepatitis B. Liver Int 2013;33(Suppl 1):111-115.

5. Singal AK, Fontana RJ. Meta-analysis: oral anti-viral agents in adults with decompensated hepatitis B virus cirrhosis. Aliment Pharmacol Ther 2012;35:674-689.

6. Mazzaro C, Dal Maso L, Urraro T, et al. Hepatitis B virus related cryoglobulinemic vasculitis: A multicentre open label study from the Gruppo Italiano di Studio delle Crioglobulinemie - GISC. Dig Liver Dis 2016;48:780-784.

7. Marcellin P, Zoulim F, Hézode C, et al. Effectiveness and safety of tenofovir disoproxil fumarate in chronic hepatitis B: A 3-year, prospective, real-world study in France. Dig Dis Sci 2016;61:3072-3083.

8. Pol S, Lampertico P. First-line treatment of chronic hepatitis B with entecavir or tenofovir in 'real-life' settings: from clinical trials to clinical practice. J Viral Hepat 2012;19:377-386.

9. Sarin SK, Kumar M, Lau GK, et al. Asian-Pacific clinical practice guidelines on the management of hepatitis B: a 2015 update. Hepatol Int 2016;10:1-98.

10. Maggi P, Montinaro V, Leone A, et al. Bone and kidney toxicity induced by nucleotide analogues in patients affected by HBVrelated chronic hepatitis: a longitudinal study. J Antimicrob Chemother 2015;70:1150-1154.

11. Han Y, Zeng A, Liao H, Liu Y, Chen Y, Ding H. The efficacy and safety comparison between tenofovir and entecavir in treatment of chronic hepatitis B and HBV related cirrhosis: A systematic review and Meta-analysis. Int Immunopharmacol 2017;42:168-175.

12. Coppolino G, Simeoni M, Summaria C, et al. The case of chronic hepatitis B treatment with tenofovir: an update for nephrologists. $J$ Nephrol 2015;28:393-402.

13. Buti M, Gane E, Seto WK, et al; GS-US-320-0108 Investigators. Tenofovir alafenamide versus tenofovir disoproxil fumarate for the treatment of patients with HBeAg-negative chronic hepatitis $B$ virus infection: a randomised, double-blind, phase 3, noninferiority trial. Lancet Gastroenterol Hepatol 2016;1:196-206.

14. Chan HL, Fung S, Seto WK, et al; GS-US-320-0110 Investigators. Tenofovir alafenamide versus tenofovir disoproxil fumarate for the treatment of $\mathrm{HBeAg}$-positive chronic hepatitis B virus infection: a randomised, double-blind, phase 3, non-inferiority trial. Lancet Gastroenterol Hepatol 2016;1:185-195.

15. Dalekos GN, Zervou E, Karabini F, Tsianos EV. Prevalence of viral markers among refugees from southern Albania: increased incidence of infection with hepatitis A, B and D viruses. Eur J Gastroenterol Hepatol 1995; 7:553-558.

16. Elefsiniotis IS, Vezali E, Brokalaki H, Tsoumakas K. Hepatitis $\mathrm{B}$ markers and vaccination-induced protection rate among Albanian pregnant women in Greece. World $J$ Gastroenterol 2009; 15:5498-5499.

17. Gikas A, Lambadiari V, Sotiropoulos A, Panagiotakos D, Pappas S. Prevalence of major cardiovascular risk factors and coronary heart disease in a sample of Greek adults: the Saronikos study. Open Cardiovasc Med J 2016;10:69-80.

18. Notara V, Panagiotakos DB, Michalopoulou M, et al; Greecs Study Investigators. Diabetes mellitus, hypertension and hypercholesterolemia in relation to the 10-year ACS prognosis; the GREECS study. Curr Vasc Pharmacol 2016;14:295-301.

19. Sfikakis PP, Bournia VK, Sidiropoulos P, et al. Biologic treatment for rheumatic disease: real-world big data analysis from the Greek country-wide prescription database. Clin Exp Rheumatol 2017;35:579-585.

20. Barmpari ME, Kokkorou M, Micheli A, et al. Thyroid dysfunction among Greek patients with type 1 and type 2 diabetes mellitus as a disregarded comorbidity. J Diabetes Res 2017;2017:6505814.

21. Loglio A, Viganò M, Grossi G, et al. Lamivudine prophylaxis prevents hepatitis $\mathrm{B}$ virus reactivation in anti-HBc positive patients under rituximab for non-Hodgkin lymphoma. Dig Liver Dis 2019;51:419-424.

22. Tian T, Song C, Jiang L, et al. Hepatitis B virus infection and the risk of cancer among the Chinese population. Int J Cancer 2020 Jun 1 [Online ahead of print]. doi: 10.1002/ijc.33130

23. Chen CL, Yang HI, Yang WS, et al. Metabolic factors and risk of hepatocellular carcinoma by chronic hepatitis B/C infection: a follow-up study in Taiwan. Gastroenterology 2008;135:111-121.

24. Elkrief L, Chouinard P, Bendersky N, et al. Diabetes mellitus is an independent prognostic factor for major liver-related outcomes in patients with cirrhosis and chronic hepatitis C. Hepatology 2014; 60:823-831.

25. Li X, Xu H, Gao P. Diabetes mellitus is a risk factor for hepatocellular carcinoma in patients with chronic hepatitis B virus infection in China. Med Sci Monit 2018;24:6729-6734.

26. Younossi Z, Kochems K, de Ridder M, Curran D, Bunge EM, de Moerlooze L. Should adults with diabetes mellitus be vaccinated against hepatitis B virus? A systematic review of diabetes mellitus and the progression of hepatitis B disease. Hum Vaccin Immunother 2017;13:2695-2706.

27. Manolakopoulos S, Striki A, Papastergiou V, et al. Persistence and adherence to nucleos $(\mathrm{t})$ ide analogues in chronic hepatitis $\mathrm{B}$ : a multicenter cohort study. Eur J Gastroenterol Hepatol 2020;32:635-641. 